BULGARIAN ACADEMY OF SCIENCES

CYBERNETICS AND INFORMATION TECHNOLOGIES • Volume 14, No 4

Sofia $\bullet 2014$

Print ISSN: 1311-9702; Online ISSN: 1314-4081

DOI: 10.1515/cait-2014-0005

\title{
Control of Traffic Driven Epidemics in Weighted Networks by Immunizing Edges
}

\author{
Zinan Chang ${ }^{1,2}$, Fei Shao ${ }^{1,2}$ \\ ${ }^{1}$ School of Computer Engineering, Jinling Institute of Technology, Nanjing 211169, China \\ ${ }^{2}$ Jiangsu Information Analysis Engineering Laboratory, Nanjing 211169, China \\ Emails: changzn@jit.edu.cn_shaofei@jit.edu.cn
}

\begin{abstract}
Many immunization strategies have been proposed to control the epidemic spreading which mainly focus on how to immunize the nodes. A novel and efficient strategy to control the traffic driven epidemic spreading in weighted networks is proposed in this paper. By immunizing the edges according to different weights, our control strategies cannot only reduce the epidemic spreading velocity and enhance the critical epidemic threshold, but also maintain the integrity of the weighted networks. Simulations show that the control strategy by immunizing edges according to the product of the strengths of two nodes of the edge proved to be more efficient.
\end{abstract}

Keywords: Traffic driven, epidemic spreading, weighted networks, SI model, SIR model.

\section{Introduction}

Outbreaks of large-scale international diseases, such as SARS, H1N1 and H5H1, have caused dramatically increasing attention in recent years. These disease outbreaks in real systems can be viewed as epidemic spreading on complex networks, the nodes representing individuals and the edges denoting the possible relationships between them. The study on complex networks has shed light on the exploration of the epidemic spreading, taking place in the network. A lot of models have been proposed to investigate the epidemic spreading in real world networks, based on the assumption, that the nodes are classified in three states: susceptible (which will not infect others, but may be infected), infected (which is infective) and recovered (which have recovered from the disease and will not be infected). The SI 
[1-3], SIS [4-6], and SIR [7-9] models are proposed based on the discrete states of the nodes. These models assume that a susceptible node will be infected by an infected one during one time step with probability $v$, while in SIS model and SIR model the infected nodes will be recovered with rate $\psi$ at the same time. Hence, the efficient spreading rate $\lambda$ is defined as $\lambda=v / \psi$. Without lack of generality, we always set $\psi=1$, since it only affects the definition of the time scale. Based on these models, a great deal of immunization strategies have been proposed [10-13] to control the epidemic spreading in real world networks, such as random immunization [10], targeted immunization [11], and acquaintance immunization [12].

These theoretical models are largely based on the hypothesis that the spreading is driven by reaction processes, in the sense that the transmission occurs from every infected node through all its neighbors at each time step. However, in some real world networks, lots of networks are found the nodes of which only interact with some of their neighbors. For instance, Internet connection at a given time depends on the specific traffic and routing protocols. Also in some other real world networks, the epidemic spreading will not occur, unless there is at least one packet interaction in the network, which can physically transfer the epidemic from one node to another even when there is an edge, linking two neighbor nodes. The epidemic spreading mainly depends on the traffic flow. A novel approach, called traffic driven epidemic spreading, is introduced to investigate the dynamic process [14-16].

The previous studies on networks have been mainly focused on the not weighted network, where the edges between nodes are either present or not. However, lots of real world networks proved to be specified not only by topology, but also by the edge weight, such as the scientific collaboration networks [17], the world-wide airport network [18], and Internet [19]. How the traffic-driven epidemic spreads in weighted networks deserves a further study. The aforementioned strategies focus on immunizing nodes to control the epidemic spreading which often isolates the nodes from the networks and breaks the integrity. In this paper we propose a new control strategy which can maintain the integrity by immunizing some specified edges to control the traffic driven epidemic spreading in weighted networks.

This paper is organized as follows. In Section 2 we describe the traffic driven epidemics spreading models and our control strategies, followed by experimental simulations and analysis in Section 3. Conclusions are proposed in Section 4.

\section{Models}

\subsection{Network model}

The BBV weighted networks can be described by a weighted adjacency matrix $\boldsymbol{W}$, whose elements $w_{i j}$ denote the weight of the edge between node $i$ and $j$. The generation of BBV weighted networks is based on the following two coupled mechanisms $[18,20]$ : 
(i) Growth. Starting from an initial seed of $N_{0}$ nodes fully connected by edges with assigned weight $w_{0}$, a new node is added at each time step. The new node will be connected to $m$ different previously existing nodes with equal weight $w_{0}$ for every edge, choosing preferentially the nodes according to the probability $\prod_{n \rightarrow i}=s_{i} / \sum_{l} s_{l}$, where $s_{i}$ is the node strength, described as $s_{i}=\sum_{j} w_{i j}$.

(ii) Weight dynamics. The weight of each new edge is initially set to a given value $w_{0}$. But the creation of the edge connecting to node $i$ will introduce variations of the weight of the other edges linked to node $i$, which is set to be proportional to the edge weights. If the total increase is $\delta$ ( $\delta_{i}=\delta$ for simplicity), we can get

$$
w_{i j}=w_{i j}+\Delta w_{i j}=w_{i j}+\delta \frac{w_{i j}}{s_{i}} .
$$

\subsection{Traffic driven epidemic spreading model}

The traffic driven epidemic spreading model can be described as given below, with the assumption that each node has unbounded packet delivery capability:

(i) all the nodes can create packets; receive packets, and forward packets;

(ii) a packet is generated at every node with probability $\beta$, with randomly selected sources and destinations; and all the packets are forwarded one step toward their destinations according to the weighted shortest path routing strategy $[17,21]$ at the same time;

(iii) a packet is removed from the network when it reaches its destination;

(iv) a susceptible node has a probability $\lambda$ of becoming infected every time it receives a packet from an infected neighbour. (in SIR model the probability $\lambda$ is the efficient spreading rate while the recovering rate is fixed to 1).

We also take advantage of the weighted betweenness $b_{w_{i}}$ to estimate the possible packets passing through node $i$. The weighted betweenness of node $i, b_{w_{i}}$, is defined as

$$
b_{w_{i}}=\sum_{s, t} \frac{\sigma(s, i, t)}{\sigma(s, t)}
$$

$\sigma(s, i, t)$ is the number of the weighted shortest paths between nodes $s$ and $t$, that pass through node $i$ and $\sigma(s, t)$ is the total number of weighted shortest paths between node $s$ and $t$.

When investigating the dynamical behaviours at the very early stage of the epidemic outbreaks, SI model is used, where the infected nodes remain always infective and spread the infection to susceptible neighbors with spreading rate $\lambda$. With the average density of infected nodes of degree $k$, defined as $i_{k}(t)$ and $s_{k}(t)$ for susceptible nodes, in BBV weighted networks we have

$$
\left\{\begin{array}{l}
\frac{d s_{k}(t)}{d t}=-\lambda \beta n b_{w_{k}} s_{k}(t) \Theta(t), \\
\frac{d i_{k}(t)}{d t}=\lambda \beta n b_{w_{k}} s_{k}(t) \Theta(t) .
\end{array}\right.
$$


The right-hand side of the second formula in (3) takes into account the probability that a node with $k$ neighbours belongs to the susceptible class $s_{k}(t)$ and gets the infection via packets travelling from infected nodes. The latter is determined by the spreading probability $\lambda$, the number of packets that a node of degree $k$ receives at each time step $\beta n b_{w_{k}}$ ( $n$ is the total node number), and the probability $\Theta(t)$ that a packet travels through an edge linking to an infected node is

$$
\Theta(t)=\frac{\sum_{k} b_{w_{k}} P(k) i_{k}(t)}{\sum_{k} b_{w_{k}} P(k)}=\frac{\sum_{k} b_{w_{k}} P(k) i_{k}(t)}{<b_{w}>} .
$$

According to the definition of $s_{k}(t)$ and $i_{k}(t)$, we have $s_{k}(t)+i_{k}(t)=1$. With the initial condition $i(t)_{t=0}=i_{0}$, we obtain the average density of infected nodes $i(t)$ :

$$
i(t)=i_{0}\left(\frac{\left\langle b_{w}\right\rangle^{2}}{\left\langle b_{w}{ }^{2}\right\rangle}\left(e^{\frac{\left.\lambda \beta n<b_{w}{ }^{2}\right\rangle}{\left\langle b_{w}>\right.} t}-1\right)+1\right) .
$$

When it turns to the SIR model, which is often used where the infected nodes get recovered and will not return to the susceptible state again, the nodes run stochastically through the cycle susceptible $\rightarrow$ infected $\rightarrow$ recovered. With the efficient spreading rate, defined as $\lambda(\psi=1)$, we have

$$
\left\{\begin{array}{l}
\frac{d s_{k}(t)}{d t}=-\lambda \beta n b_{w_{k}} s_{k}(t) \Theta(t), \\
\frac{d i_{k}(t)}{d t}=-i_{k}(t)+\lambda \beta n b_{w_{k}} s_{k}(t) \Theta(t), \\
\frac{d r_{k}(t)}{d t}=i_{k}(t),
\end{array}\right.
$$

$r_{k}(t)$ means the average density of the recovered nodes of degree $k$.

By imposing $d i_{k}(t) / d t=0$ and $s_{k}(t)+i_{k}(t)+r_{k}(t)=1$, we can obtain the epidemic threshold of the traffic driven SIR epidemic model:

$$
\lambda_{\mathrm{c}}=\frac{<b_{w}>}{<b_{w}{ }^{2}>\beta n} .
$$

The epidemic will outbreak when the spreading rate $\lambda>\lambda_{\mathrm{c}}$.

\subsection{Control strategy}

We give our control strategies as follows:

(i) Define the weight of the edge linking two nodes $i$ and $j, W_{i j}$, in a different way according to the different control strategies, as shown in Table 1.

(ii) Sort the edges by their definition weights $W_{i j}$ in a decreasing order and immunize the edge ranked first. If immunizing an edge will cause some nodes to be isolated from the network, we will not immunize it and will deal with the edge ranked next.

(iii) Recalculate the definition weight $W_{i j}$ and repeat step (ii) until a fraction $f_{\mathrm{e}}$ of the edges is immunized. 
Table 1. The definition weight $W_{i j}$ of different control strategies

\begin{tabular}{|c|c|c|c|}
\hline $\begin{array}{c}\text { Control } \\
\text { strategy }\end{array}$ & DOW & DDP & DSP \\
\hline$W_{i j}$ & $w_{i j}$ & $k_{i} k_{j}$ & $s_{i} s_{j}$ \\
\hline
\end{tabular}

We utilize the RAN immunization strategy, which randomly immunizes edges, to check the validity of our immunization strategies.

There must be at least $n-1$ edges to maintain the integrity of the network. Therefore, $f_{\mathrm{e}}=1$ means that about $n m-n+1$ edges are immunized.

\section{Simulations and analysis}

At first, we test the validity of our control strategies on SI model by checking the average density of the infected nodes, using BBV weighted networks with $n=100$, $\delta=4, m=4$ and $\omega_{0}=1$. The simulation results are shown in Fig. 1 .

(For every network, 20 instances are generated and for each instance, we run 50 simulations. The results are the average over all simulations.)
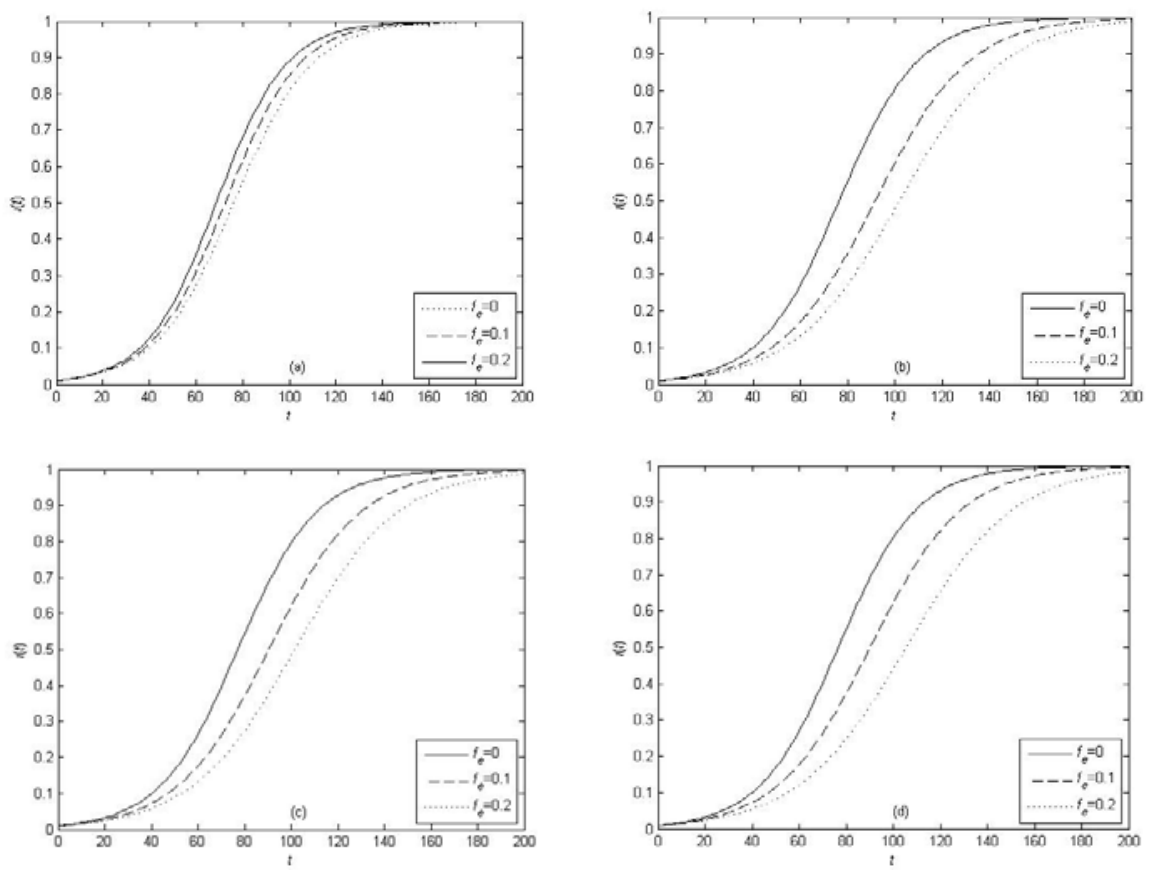

Fig. 1. Average density of the infected nodes $i(t)$ versus time $t$ in a BBV network with $n=100, \delta=4$, $m=4, \omega_{0}=1, \Lambda=0.005, \beta=1$. RAN (a); DOW (b); DDP (c); DSP (d)

As shown in Fig. 1, we can consider that immunizing some specified edges can reduce the epidemic spreading velocity, which can be measured by the average density of the infected nodes, while randomly immunizing edges cannot. The average density of infected nodes of the DSP control strategy is the lowest after immunizing $10 \%$ or $20 \%$ of the edges. It means that DSP control strategy, which 
immunizes edges with higher product of the strengths of two nodes of the edge is more efficient in controlling the traffic driven epidemic spreading.

Then we turn to SIR model and immunize $f_{\mathrm{e}}=1$ edges to validate our control strategies by examining the critical epidemic threshold $\lambda_{\mathrm{c}}$.

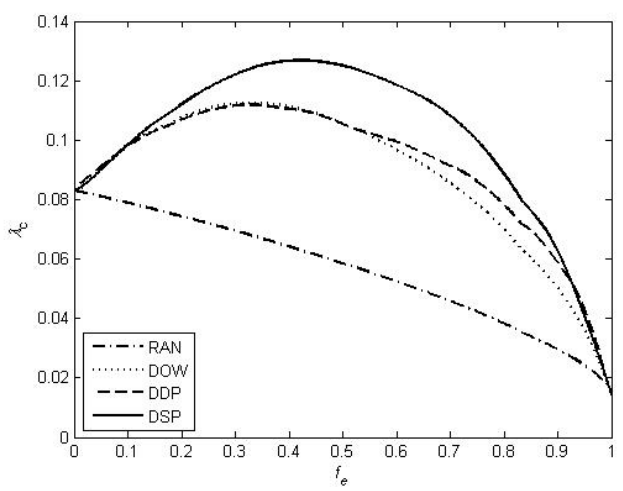

Fig. 2. Relationship $\lambda_{\mathrm{c}}\left(f_{\mathrm{e}}\right)$ : BBV network with $n=100, \delta=4, m=4, \omega_{0}=1, \beta=1$

Fig. 2 indicates the relationship of the critical epidemic threshold $\lambda_{\mathrm{c}}$ and the fraction of immunized edges $f_{\mathrm{e}}$. The DSP control strategy which immunizes edges with higher product of the strengths of two nodes of the edge obtains the maximum epidemic threshold. The maximum epidemic threshold of DSP control strategy is $52.17 \%$ higher than that of the original network $\left(f_{\mathrm{e}}=0\right)$, while the DDP control strategy and the DOW control strategy is $33.40 \%$ and $35.46 \%$ correspondingly. The maximum epidemic threshold of the DSP control strategy is better than of the other two strategies (14.08\% higher than DDP and $12.33 \%$ higher than DOW). It also proves that the DSP control strategy is more efficient to control the traffic driven epidemic spreading than the other control strategies. The epidemic threshold presents an inverse $\mathrm{U}$ shape which always goes up, when $f_{\mathrm{e}}$ is less than 0.5 and declines when more edges are immunized. When $f_{\mathrm{e}}$ is 1 , the number of edges is almost equal to the number of nodes and the network will be modified to a treelike topology. The epidemic can become endemic even at a very small spreading rate.

In order to check the impact, the weight on the critical epidemic threshold $\lambda_{\mathrm{c}}$, we change $\delta$ to 8 to get the respective results, shown in Fig. 3.

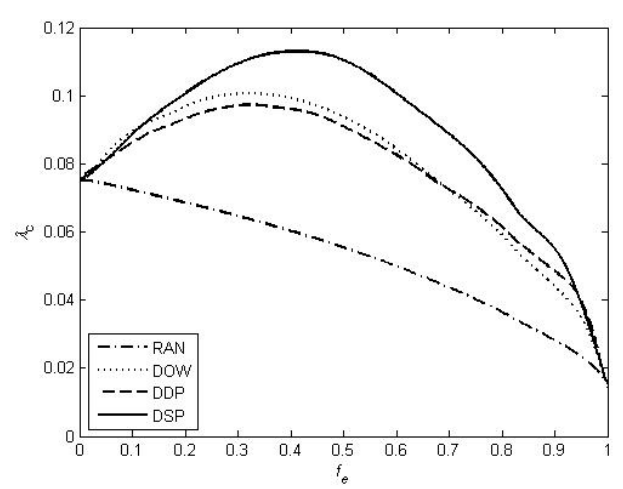

Fig. 3. Relationship $\lambda_{\mathrm{c}}\left(f_{\mathrm{e}}\right)$ : BBV network with $n=100, \delta=8, m=4, \omega_{0}=1, \beta=1$ 
Fig. 3 shows that the enhancement of three control strategies is $49.58 \%$, $28.81 \%$ and $33.30 \%$ respectively, which means that the total increase weight $\delta$ nearly does not affect our control strategies.

Then we examine the implication of the new added edge number $m$ on our control strategies by setting $m$ to 8 and obtain the simulations in Fig. 4.

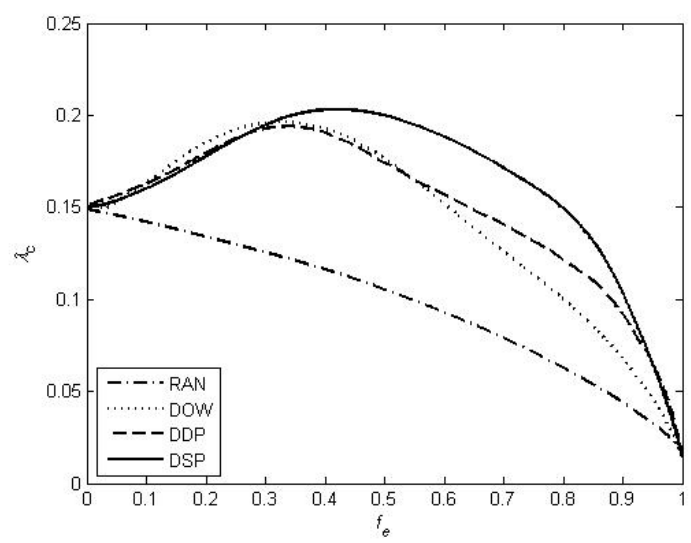

Fig. 4. Relationship $\lambda_{\mathrm{c}}\left(f_{\mathrm{e}}\right)$ : BBV network with $n=100, \delta=4, m=8, \omega_{0}=1, \beta=1$

Fig. 4 shows almost the same result, as Figs 2 and 3. The enhancement of three routing strategies is $35.97 \%, 29.64 \%$ and $31.31 \%$ correspondingly, which means the more new added edges do not also affect our control strategies. And we can also find that deleting edges randomly cannot restrain the traffic driven epidemic spreading.

Then we check the impact of the node number $n$ to gain the results in Fig. 5.

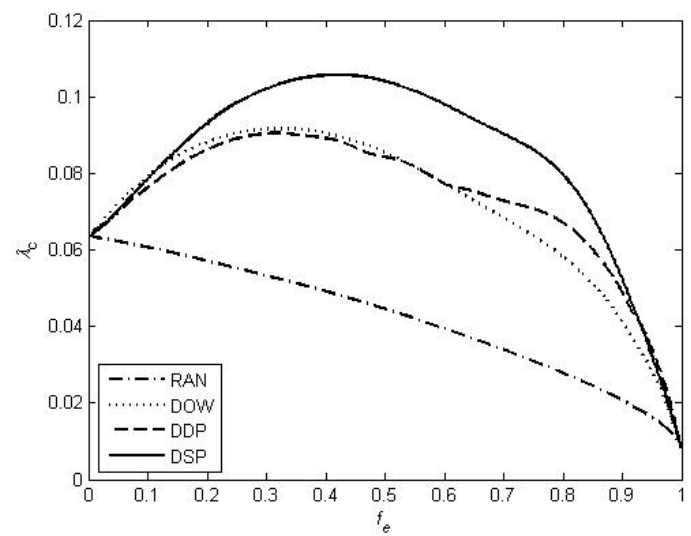

Fig. 5. Relationship $\lambda_{\mathrm{c}}\left(f_{\mathrm{e}}\right)$ : BBV network with $n=200, \delta=4, m=4, \omega_{0}=1, \beta=1$

The enhancement of the three routing strategies (DSP, DDP, and DOW) is $65.65 \%, 41.36 \%$ and $43.82 \%$ correspondingly, which are higher than that shown in Fig. 2. It indicates that our control strategies are more efficient in large scale networks.

Finally, we check the impact of the traffic flow on our control strategies. We generate packets at every node with probability $\beta=2$. 


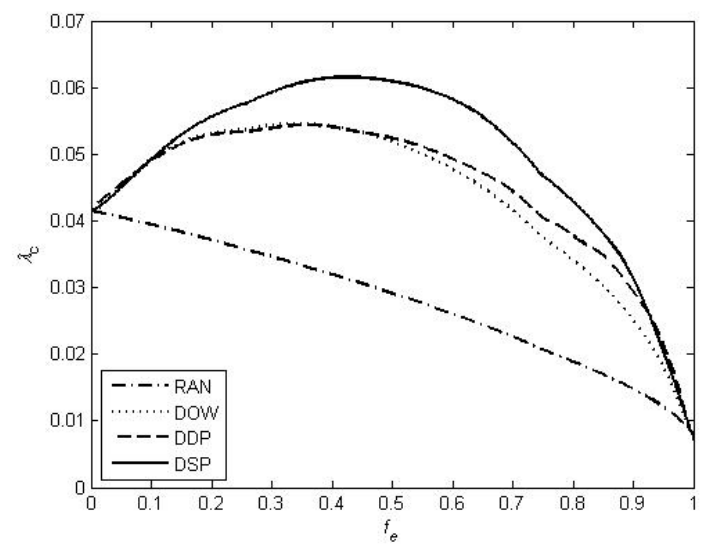

Fig. 6. Relationship $\lambda_{\mathrm{c}}\left(f_{\mathrm{e}}\right)$ : BBV network with $n=100, \delta=4, m=4, \omega_{0}=1, \beta=2$

Comparing Fig. 6 with Fig. 2, we can observe that the two figures are almost the same, except the absolute value of the critical epidemic threshold $\lambda_{\mathrm{c}}$. When the packet generating probability is doubled, there are more packets transferring across the network which will result in increase of the epidemic spreading velocity. Therefore, the critical epidemic threshold is doubled.

Finally, we test our control strategies on USAir 97 network (network of direct flight connections between US airports for the year 1997 with 332 nodes and 2126 edges, http://vlado.fmf.uni-lj.si/pub/networks/data/). We immunize $20 \%$ of the edges and check the average density of the infected nodes of SI model and the critical epidemic threshold of SIR model to confirm our control strategies.

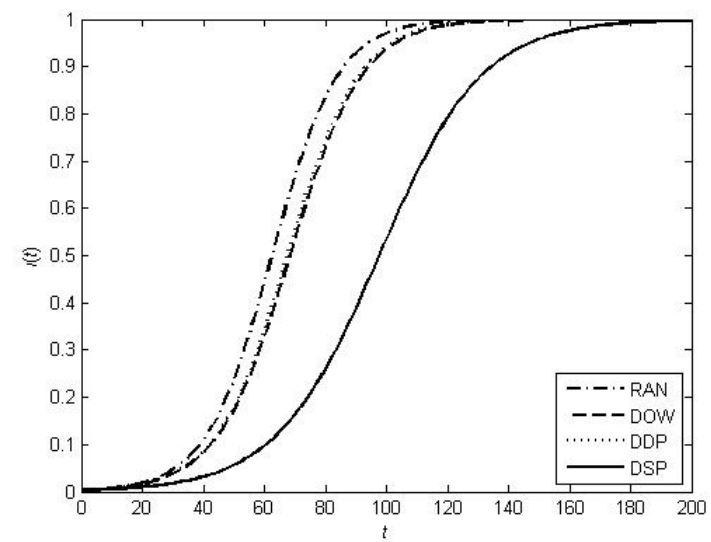

Fig. 7. Relationship $i(t)(t)$ in USAir 97 network, $f_{\mathrm{e}}=0.2, \lambda=0.001, \beta=1$

From Fig. 7 we can realize that after immunizing $f_{\mathrm{e}}=0.2$ edges in real world network, the average density of the infected nodes of DSP control strategy is still the lowest. The maximum epidemic threshold of the DSP control strategy is 0.020 which is $80.39 \%$ higher than that of the original network (and for DDP, DOW control strategies it is $44.56,46.85 \%$ respectively.) It proves that DSP control 
strategy which immunizes the edges with a higher product of the strengths work also well in a real world network.

\section{Conclusion}

Aiming at restraining the epidemic spreading to the greatest possible extent, we propose novel immunization strategies of immunizing some specified edges to maintain the integrity of the network. By examining the epidemic spreading velocity of SI model and the critical epidemic threshold of SIR model, the simulations show that the strategies proposed can efficiently control the traffic driven epidemic spreading in BBV networks. The DSP control strategy which immunizes edges with higher product of the strengths of two nodes of the edge proved to be more efficient. A transition is found at the critical point after immunizing about half of the edges. Our control strategies can maintain the integrity of the whole network which makes it more appropriate in some particular situations. Our control strategies may be of great significance for controlling the epidemic spreading in real world networks. There may be other definitions of the weight of the edge and more suitable definitions of the weight will be a problem deserving further study.

Acknowledgements: This work was partially supported by the National Natural Science Foundation of China (Grant No 61373136, 61375121), the Natural Science Foundation of Jiangsu Province, China (Grant No BK2012082), the Natural Science Fund for Colleges and Universities in Jiangsu Province, China (Grant No 14KJB520012), the Research Foundation of Jinling Institute of Technology (Grant No JIT-B-201406) and sponsored by Qing Lan Project and Jiangsu Provincial Government Scholarship for Overseas Studies.

\section{References}

1. B a r th é le m y, M., et al. Velocity and Hierarchical Spread of Epidemic Outbreaks in Scale-Free Networks. - Physical Review Letters, Vol. 92, 2004, No 17, 178-701.

2. B a r t h e 1 e m y, M., et al. Dynamical Patterns of Epidemic Outbreaks in Complex Heterogeneous Networks. - Journal of Theoretical Biology, Vol. 235, 2005, No 2, 275-288.

3. Z $\mathrm{hou}, \mathrm{T}$., et al. Behaviors of Susceptible-Infected Epidemics on Scale-Free Networks with Identical Infectivity. - Physical Review E, Vol. 74, 2006, No 5, 56-109.

4. Pas tor-S a torras, R., A. Vespignani. Epidemic Spreading in Scale-Free Networks. Physical Review Letters, Vol. 86, 2001, No 14, 3200-3203.

5. Pastor-S at orras, R., A. Vespignani. Epidemic Dynamics and Endemic States in Complex Networks. - Physical Review E, Vol. 63, 2001, No 6, 66-117.

6. Heth c ote, H. W. The Mathematics of Infectious Diseases. - SIAM Review, Vol. 42, 2000, No 4, 599-653.

7. M a y, R. M., A. L. L 1 o y d. Infection Dynamics on Scale-Free Networks. - Physical Review E, Vol. 64, 2001, No 6, 66-112.

8. Moreno, Y., R. Pastor-S at orras, A. Vespignani. Epidemic Outbreaks in Complex Heterogeneous Networks. - European Physical Journal B, Vol. 26, 2002, No 4, 521-529.

9. B a il e y, N. T. J. The Mathematical Theory of Infectious Diseases and its Applications. New York, Hafner Press, 1975.

10. A nders o n, R. M., R. M. M a y, B. A n d ers o n. Infectious Diseases of Humans: Dynamics and Control. Oxford, Oxford University Press, 1992. 
11. P a s t o r-S a t o rra s, R., A. V e s p i g n a n i. Immunization of Complex Networks. - Physical Review E, Vol. 65, 2002, No 3, 36-104.

12. C o h e n, R., S. H a v li n, D. B e n-A v r a h a m. Efficient Immunization Strategies for Computer Networks and Populations. - Physical Review Letters, Vol. 91, 2003, No 24, $247-901$.

13. Zhang, H. F., et al. An Efficient Control Strategy of Epidemic Spreading on Scale-Free Networks. - Chinese Physics Letters, Vol. 26, 2009, No 6, 68-901.

14. Meloni, S., A. Arenas, Y. Moren o. Traffic-Driven Epidemic Spreading in Finite-Size Scale-Free Networks. - PNAS, Vol. 106, 2009, No 40, 16897-16902.

15. Y a n g, H. X., W. X. W a ng, Y. C. La i. Traffic-Driven Epidemic Outbreak on Complex Networks: How Long Does it Take? - CHAOS, Vol. 22, 2012, No 4.

16. Y ang, H. X., et al. Traffic-Driven Epidemic Spreading on Networks of Mobile Agents. Europhysics Letters, Vol. 98, 2012, No 6.

17. N e w m a n, M. E. J. Scientific Collaboration Networks. II. Shortest Paths, Weighted Networks, and Centrality. - Physical Review E, Vol. 64, 2001, No 1, 16-132.

18. B a r r a t, A., et al. The Architecture of Complex Weighted Networks. - PNAS, Vol. 101, 2004, No $11,3747-3752$.

19. Pastor-S at orras, R., A. Vespignani. Evolution and Structure of the Internet: A Statistical Physics Approach. Cambridge, Cambridge University Press, 2007.

20. B arrat, A., M. B arthélemy, A. Vespignani. Modeling the Evolution of Weighted Networks. - Physical Review E, Vol. 70, 2004, No 6, 66-149.

21. Brandes, U. A Faster Algorithm for Betweenness Centrality. - Journal of Mathematical Sociology, Vol. 25, 2001, No 2, 163-177. 